\title{
Electrical Resonance Circuits as Analogs to Quantum Mechanical Billiards
}

\author{
K.-F. Berggren, J. Larsson and O. Bengtsson \\ Department of Physics and Measurement Technology \\ Linköping University, 58183 Linköping, Sweden
}

\begin{abstract}
We propose that a two-dimensional electric network may be used for fundamental studies of wave function properties, transport, and related statistics. Using Kirchhoff's current law and the $j \omega$-method we find that the network is analogous to a discretized Schrödinger equation for quantum billiards and dots. Thus the complex electric potentials play the role of quantum mechanical wave functions.
\end{abstract}

PACS numbers: 05.45.Mt, 73.63.Kv, 84.30.-r, 89.20.--a

\section{Introduction}

The use of analog systems to investigate the foundations of quantum mechanics is a lively field of research. Thus various types of billiards have been used for experimental studies of quantum chaos, wave function morphology, current statistics, vortex formation, and other topological issues. An advantage in going from the quantum mechanical (QM) mesoscopic to classical macroscopic systems is that experimental conditions may be controlled precisely $[1,2]$ and one may readily observe eigenstates, both their amplitude and phase, currents, etc. in a way that at present appears impossible for nanosized quantum billiards. For these reasons planar microwave cavities have been studied experimentally, for example, as in Refs. [3-7]. Figure 1 shows experimental results from Ref. [3] for a flat, effectively two-dimensional microwave cavity in the shape of an open quantum dot with "two leads". In this case the stationary Helmholtz equation for the perpendicular electric field $E$ with wave number $k$,

$$
\left(\nabla^{2}+k^{2}\right) E=0
$$

coincides with the time-independent Schrödinger equation for a hard-walled quantum billiard [1]. We may therefore say that the intensity of the electric field, $|E|^{2}$, mimics the quantum probability $\rho=|\psi|^{2}$ associated with a quantum state $\psi$. In the same way the Poynting vector emulates the quantum mechanical probability 

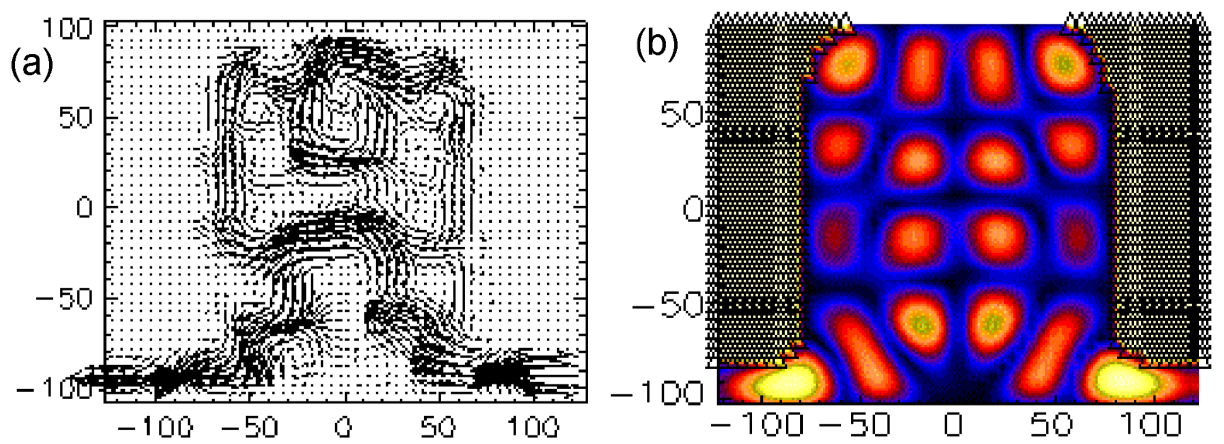

Fig. 1. Experimental results from Ref. [3] for a flat microwave cavity in the shape of an open quantum dot with "two leads". Part (a) shows the Poynting vector to be compared with the quantum mechanical probability current. Part (b) refers to the intensity of the field to be compared with the quantum probability $\rho=|\psi|^{2}$. Numbers on the axes refer to grid points.

current. Hence, micro- and matter waves in billiards are in this case expected to behave in the same manner. There are also other classical wave analogs for example acoustics, electromechanical systems, and surface waves in water vessels with arbitrary shape [1].

Here we propose another kind of emulation of quantum billiards based on electrical networks, which, if realized, should offer new and rich experimental possibilities. The idea behind our choice is the following. In numerical simulations of quantum billiards one often relies on the finite difference method [8]. This implies that a computational grid $(i, j)$ is generated in the billiard, and an equation is formed in each such numerical grid point. Usually, only nearest neighbor interactions are considered. For QM billiards this results in the five point approximation of the Schrödinger equation

$$
\psi_{i, j-1}+\psi_{i-1, j}+\psi_{i, j+1}+\psi_{i+1, j}-4 \psi_{i, j}+k^{2} \psi_{i, j}=0,
$$

where $\psi_{i, j}$ is the wave function at grid point $(i, j)$. The wave number is $k=\sqrt{2 m a^{2} \mathcal{E}} / \hbar$, where $\mathcal{E}$ is the energy eigenvalue, $a$ the distance between nearest neighbors, and $m$ the particle mass. The discretized form in Eq. (2) now suggests that various types of lattice analogs to quantum billiards may be conceived. An obvious candidate is a mechanical system with springs and masses. However, Eq. (2) is also of the same form as the tight-binding model for a lattice of resonating monoatoms. We may therefore look for a discrete lattice constructed from identical objects with some characteristic oscillatory behavior. We propose that such objects could be resonant electric circuits*. We also suggest that such sys-

${ }^{*}$ For a preliminary discussion about closed billiards see [9]. Equivalent electric circuits to represent the Schrödinger equation were actually discussed by Kron [10] already in 1945. Later Manolache and Sandu [11] have investigated the eigenmodes of closed symmetric cavities. Statistical aspects are raised in the recent work by Bulgakov et 
tems offer, in principle, new and rich possibilities for experimental studies wave functions and, in particular, wave function statistics [1-3, 14], vortex distributions $[6,15-18]$, current statistics $[3,6,19,20]$, long-range correlations and phase rigidity $[21,22]$, and current flow in the form of "quantum percolation" [23]. The selected articles give numerous references also to other relevant articles in the field. In particular we note that the electric networks discussed in this article have obvious similarities with quantum graphs [24, 25].

\section{An electrical network model}

An electrical grid is designed according to Fig. 2. The grid consists of capacitances $C$, inductances $L$, and resistances $R$. The latter are used for modeling the resistance of the inductors used in the practical case. Here, we study a grid shaped geometrically as in Fig. 3. This is the same billiard used in the microwave studies in Refs. [3-6], which originally was chosen to study wave function scarring

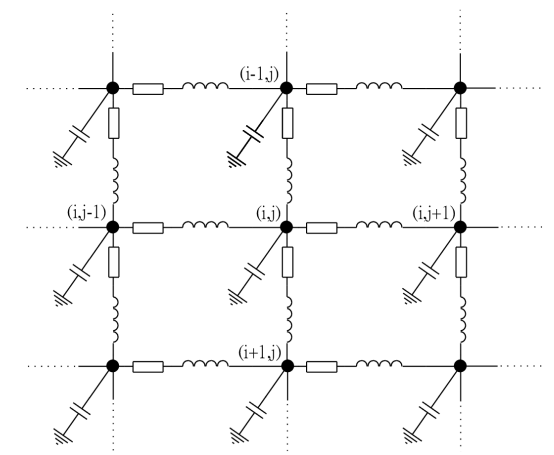

Fig. 2. Internal region of the electric grid (from Ref. [13]).

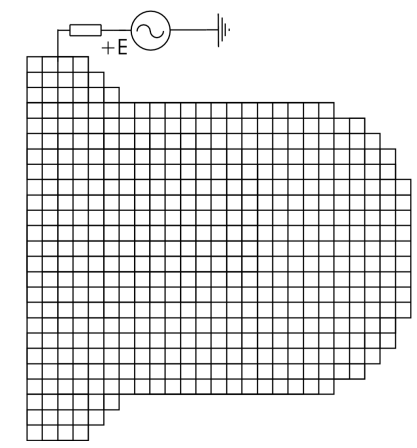

Fig. 3. Modeling of a "two-lead" cavity with a driving voltage $E$ in the shape of an open quantum dot.

al. [12], whereas Bengtsson et al. [13] have focused on wave function statistics and how symmetry may be probed by external AC voltages. References [12] and [13] are therefore supplementary. The present article summarizes our previous work. 
in a quantum dot with two leads [26]. This geometry is suitable since there are both experimental $[1,3-5]$ and QM [27] results to compare with. Because of the irregular shape we expect chaotic modes.

Let one or more sinusoidal voltages be attached to the net. Using Kirchhoff's current law and the $j \omega$-method [28], an equation in each grid point can be formed. After rearranging the terms, equations of the form

$$
-\left(V_{i, j-1}+V_{i-1, j}+V_{i, j+1}+V_{i+1, j}-4 V_{i, j}\right)=-\frac{Z_{l}}{Z_{c}} V_{i, j}
$$

are obtained, where $Z_{l}=\mathrm{j} \omega L+R, Z_{c}=1 / \mathrm{j} \omega C$, and $\mathrm{j}=\sqrt{-1}$. This equation is obviously of the same form as the discretized Schrödinger equation in expression (2). Due to the resistances $R$ there is dissipation of energy in the system. The Dirichlet boundary conditions are imposed by simply grounding the boundary grid points.

\section{Resonant modes}

Network modes, corresponding to eigenstates in QM, may be found in two ways. The first way is by assuming that the network is isolated, i.e., there are no external inputs such as voltages. Assuming that $R$ is small and may be neglected we then rewrite the system of equations in Eq. (3) in matrix form as

$$
\mathcal{B} \boldsymbol{V}=L C \omega^{2} \boldsymbol{V} .
$$

Hence,

$$
\omega=\sqrt{\frac{\operatorname{Eig}(\mathcal{B})}{L C}}
$$

gives the angular frequencies for the resonant modes and the eigenvectors of the amplitudes $V_{i, j}$. The other, more practical way of finding the eigenmodes is by examining the current through the resistance in series with the applied voltage as a function of the angular frequency. It turns out that the modes are found at the minima of the $I-\omega$ curve, compared to microwave cavities for which the modes

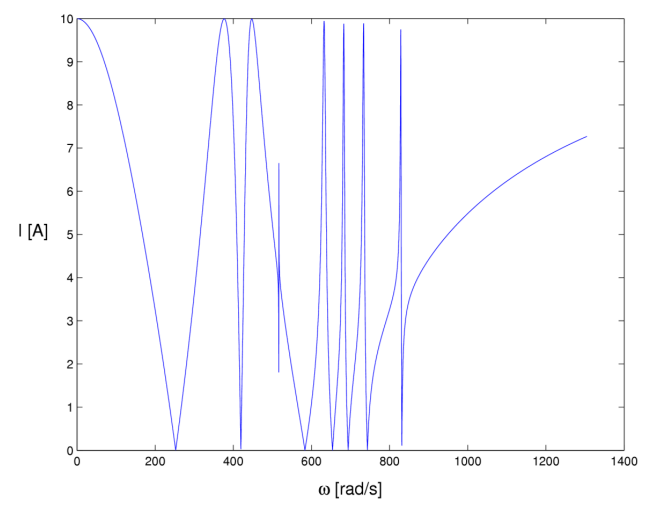

Fig. 4. The absolute value of the current as a function of the angular frequency, for a very small net. Each minimum holds an eigenmode (from Ref. [13]). 

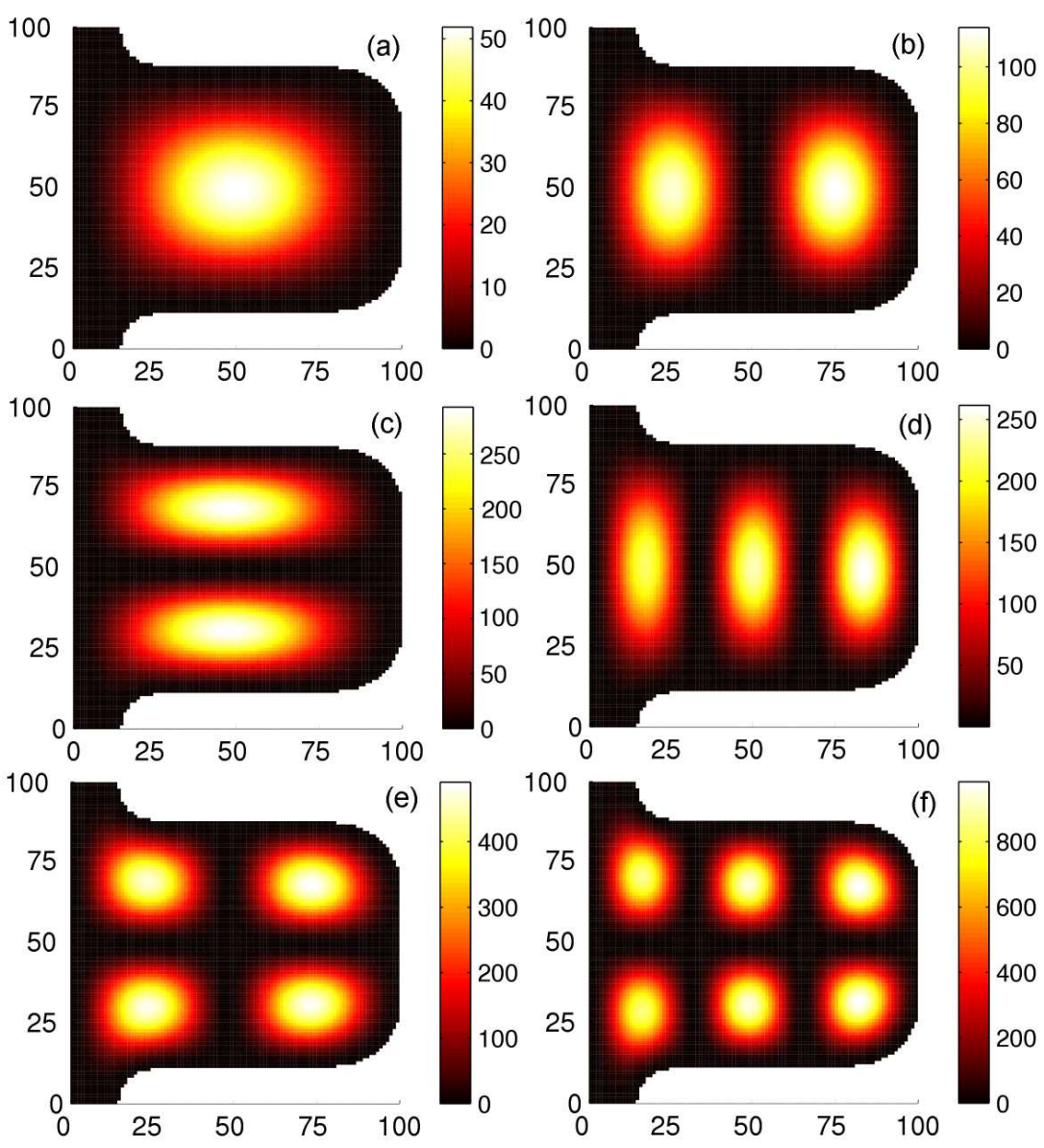

Fig. 5. The lowest six modes, showing $|V|^{2}$, to be compared to $|\psi|^{2}$ in QM. The number of grid points is $100 \times 100$, and the values $L=10 \mathrm{mH}, C=1 \mathrm{mF}$ and $R=0.05 \mathrm{~m} \Omega$ were used in the simulations.

are found at the transmission maxima [3, 4]. A typical current for a very small net is seen in Fig. 4, and the first six modes in the two-lead cavity are shown in Fig. 5. Comparisons with microwave measurements [3, 4] and QM calculations [27] verify that $|V|^{2}$ indeed mimics $|\psi|^{2}$.

\section{Current flow}

To explore the correspondence between $\psi$ and $V$ further we consider the transmission through the system. An analogue to the quantum mechanical probability current

$$
\boldsymbol{j}=\frac{\hbar}{m} \operatorname{Im}\left(\psi^{*} \nabla \psi\right)
$$

is obtained by replacing the wave function $\psi$ with our potential field $V$. Hence, 

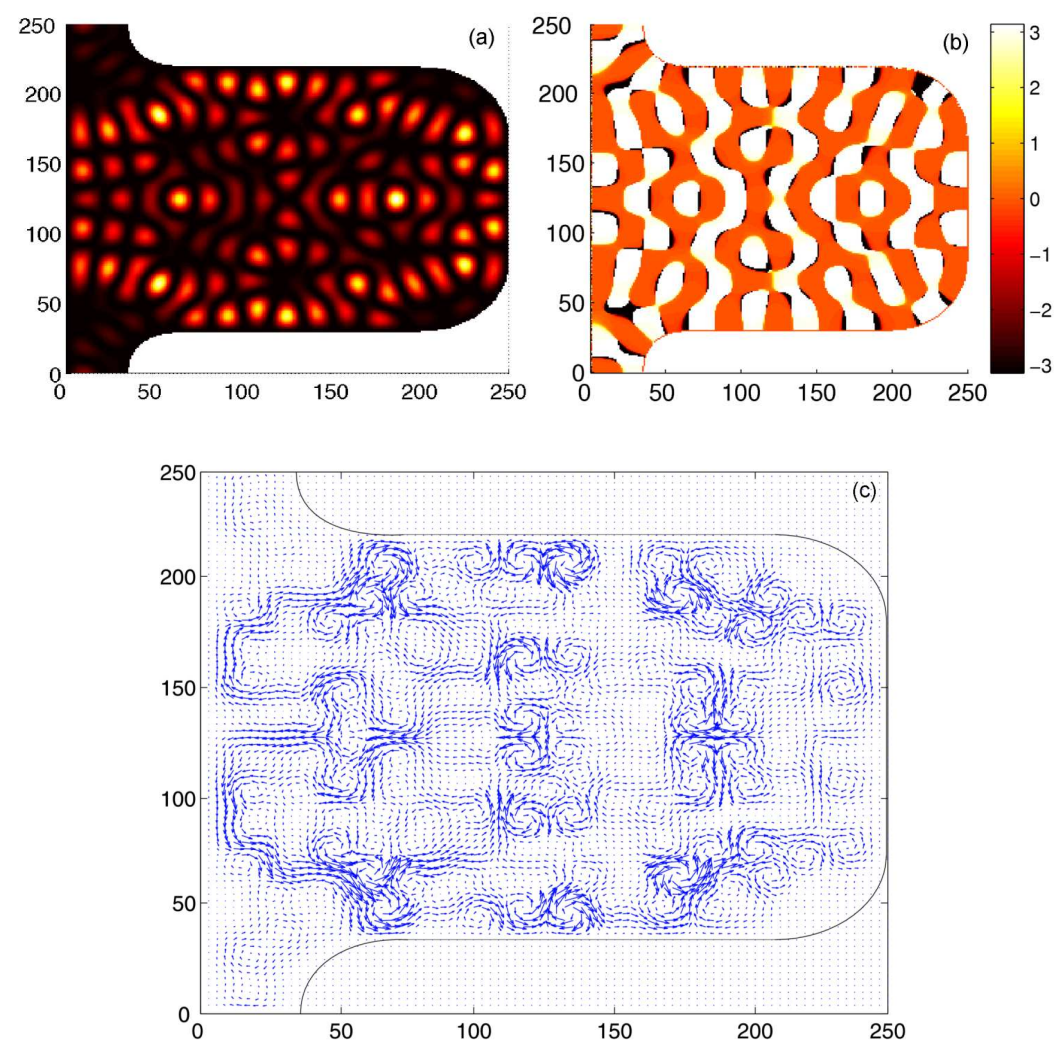

Fig. 6. A mode at higher frequency (mode number 175), with corresponding phase and the Poynting vector plots. $250 \times 250$ grid points. (a) $\left|V^{2}\right|$ for mode number 175 . (b) Phase plot, ranging from $-\pi$ to $\pi$. (c) Poynting vector field.

omitting constants we have

$$
\boldsymbol{S}=\operatorname{Im}\left(V^{*} \nabla V\right)
$$

which in fact is a Poynting vector for our system. For a single driving voltage as in Fig. 3 the computed $\boldsymbol{S}$ lacks symmetry, especially at low frequencies. However, as the frequency is increased, this feature becomes less prominent, and the plots generally coincide closely with those from QM billiards [27] and microwave cavities [3-5]. A higher frequency mode, with corresponding phase plot and Poynting vector field, is shown in Fig. 6.

\section{Symmetries and statistics}

Application of additional voltages with different phases is a useful tool for extracting states belonging to a certain symmetry class. This is essential for many statistical computations, since statistics often are only viable for an ensemble of modes belonging to a certain class of symmetry [2]. The two-lead cavity has two classes of symmetry consisting of even or odd wave functions. By connecting an 
additional voltage at the other lead one of the classes is suppressed by means of superposition of the driving voltages.

However, an easier way of identifying the modes belonging to a certain symmetry is by desymmetrizing the billiard, followed by extracting the eigenvalues of the Hamiltonian-like matrix $\mathcal{B}$. In the case of the two-lead cavity in Fig. 3, this is accomplished by imposing the Dirichlet boundary conditions along the symmetry line in the middle of the billiard, and then studying one of the halves. This procedure selects the odd wave functions.

There is a number of statistical properties derived for QM systems $[1,2,14,19]$. Some of them, concerning chaos and time-reversal symmetry (TRS), are examined here. Ideally, our system should follow the same statistics as the quantum mechanical system we intend to emulate.

A fundamental statistical property concerns the distribution of normalized spacings $s$ between eigenenergies for closed systems. As shown above, $\omega^{2}$ corresponds to the QM eigenlevel $E$. We have therefore extracted the spacings from the eigenvalues of $\mathcal{B}$ in Eq. (4). For an irregular system, such as the cavity examined here, the Wigner-Dyson distribution

$$
P(s)=\frac{\pi s}{2} \mathrm{e}^{-\pi s^{2} / 4}
$$

is expected, because $R$ is assumed negligible. Equation (8) is indeed well satisfied as shown in Fig. 7. There is also QM statistics derived for $|\psi|^{2}, \boldsymbol{j}, j_{x}$, and $j_{y}$ for individual modes. Here, the mode in Fig. 6a is examined. The state is expected to have effectively TRS, since $\operatorname{Re}(V) \gg \operatorname{Im}(V)$ at the $I-\omega$ minima $[14,19]$.

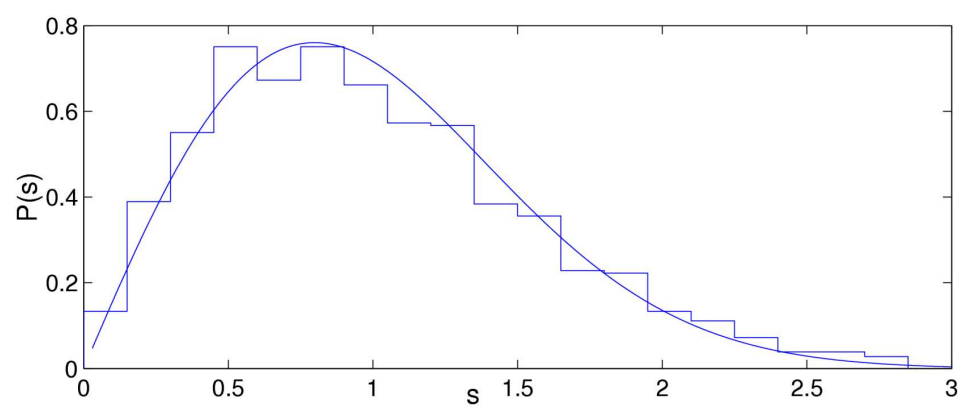

Fig. 7. Histogram showing the distribution of eigenvalue spacings for 1200 states. The solid line is the Wigner-Dyson distribution from Eq. (8) (from Ref. [13]).

In QM a plot $P(\rho)$ may be produced, where $\rho=A|\psi|^{2}$ and $A$ the area of the billiard, giving the probability of finding a certain intensity. $P(\rho)$ follows the well known Porter-Thomas distribution, given by

$$
P(\rho)=\frac{1}{\sqrt{2 \pi \rho}} \mathrm{e}^{-\rho / 2}
$$

for QM chaotic modes which effectively display TRS [1]. 
The QM probability current also follows certain statistics [19, 20]. One may study the absolute value as well as the components. For a chaotic mode the absolute value follows:

$$
P(|\boldsymbol{j}|)=\frac{|\boldsymbol{j}|}{\tau^{2}} K_{0}\left(\frac{|\boldsymbol{j}|}{\tau}\right),
$$

where $K_{0}$ is the modified Bessel function of the second kind, zeroth order, and $\tau$ is proportional to the product $\left\langle(\operatorname{Re} \psi)^{2}\right\rangle\left\langle(\operatorname{Im} \psi)^{2}\right\rangle[19]$. Here $\tau$ is treated as a parameter, used merely to see if the statistics follow the generic form predicted by theory. If the net current is small, the components should obey

$$
P\left(j_{d}\right)=\frac{1}{2 \tau} \mathrm{e}^{-\left|j_{d}\right| / \tau}
$$

where $d$ indicates horizontal or vertical direction.
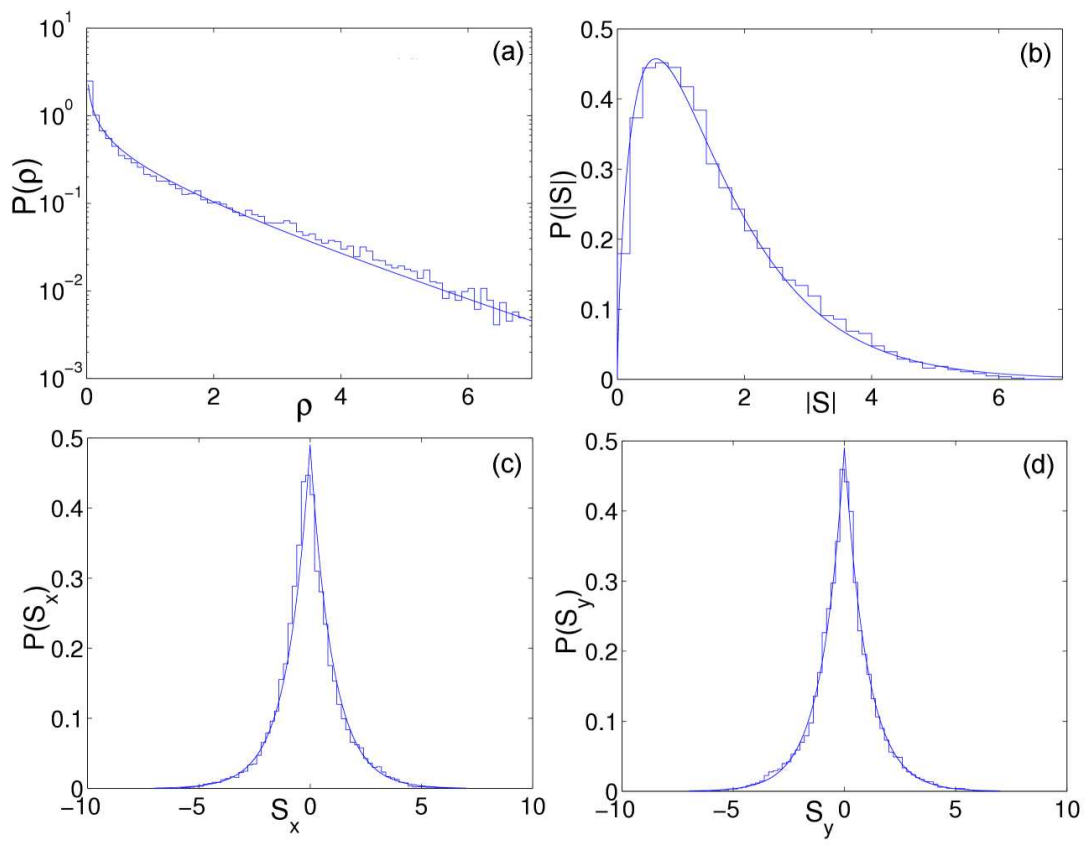

Fig. 8. Statistical properties for the mode in Fig. 6. (a) Distribution of amplitudes. (b) Statistics for $|\boldsymbol{S}|$. (c) Statistics for $S_{x}$. (d) Statistics for $S_{y}$. The solid line in (a) is the Porter-Thomas distribution, given by Eq. (9). The solid lines in (b)-(d) are given by Eq. (10) and Eq. (11) with $\tau=1.02$.

Figure 8 shows histograms for distributions related to the mode in Fig. 6a with $\psi$ replaced by $V$ and $\boldsymbol{j}$ by $\boldsymbol{S}$. The numerical results for our electric network obviously agree nicely with theoretical predictions for random fields. 


\section{Summary}

We have proposed that electrical networks, if practicable, could be used for fundamental studies of wave function properties and transport in general and, more specifically, their mapping onto open quantum dots. By connecting each grid point to some light source, using transistors, probes, etc., the wave patterns could be observed in real time. The role of dissipation and breaking of TRS may also be studied in a controlled way via the resistance $R$. One could also model any billiard, it is only a question of grounding certain grid points. In addition to the scientific case our network has obvious pedagogical merits.

\section{Acknowledgments}

We acknowledge discussions with Almas Sadreev about common issues, with Jani Hakanen about computational problems, and with Lars Wanhammar about electric circuits.

\section{References}

[1] H.-J. Stöckmann, Quantum Chaos: An Introduction, Cambridge University Press, Cambridge 1999, ISBN 0-521-59284-4 and references therein.

[2] T. Guhr, A. Müller-Groeling, H.A. Weidenmüller, Phys. Rep. 299, 189 (1998) and references therein.

[3] M. Barth, Ph.D. thesis, Philipps-Universität, Marburg 2001.

[4] Y.-H. Kim, M. Barth, H.-J. Stöckmann, J.P. Bird, Phys. Rev. B 65, 165317 (2002).

[5] M. Barth, H.-J. Stöckmann, Phys. Rev. E 65, 66208 (2002).

[6] Y.-H. Kim, M. Barth, U. Kuhl, H.J. Stöckmann, Prog. Theor. Phys. Suppl. 150, 106 (2003).

[7] C. Dembowski, H.D. Gräf, H.L. Harney, A. Heine, W.D. Heiss, H. Rehfeld, A. Richter, Phys. Rev. Lett. 86, 787 (2001).

[8] F.J. Vesely, Computational Physics: An Introduction, Plenum Press, New York 1994.

[9] K.-F. Berggren, A.F. Sadreev, in: Proc. Conf. Mathematical Modelling of Wave Phenomena, Växjö 2002, Eds. B. Nilsson, L. Fishman, in Mathematical Modelling in Physics, Engineering and Cognitive Sciences, Vol. 7, Växjö University Press, Växjö 2004, p. 229, ISBN 91-7636-385-6, ISSN 1651-0267.133-147.

[10] G. Kron, Phys. Rev. 67, 39 (1945).

[11] F. Manolache, D.D. Sandu, Phys. Rev. A 49, 2318 (1994).

[12] E.N. Bulgakov, D.N. Maksimov, A.F. Sadreev, Phys. Rev. E 71, 046205 (2005).

[13] O. Bengtsson, J. Larsson, K.-F. Berggren, Phys. Rev. E 71, 056206 (2005).

[14] H. Ishio, A.I. Saichev, A.F. Sadreev, K.-F. Berggren, Phys. Rev. E 64, 56208 (2001).

[15] N. Shvartsman, I. Freund, Phys. Rev. Lett. 72, 1008 (1994).

[16] A.I. Saichev, K.-F. Berggren, A.F. Sadreev, Phys. Rev. E 64, 036222 (2001). 
[17] K.-F. Berggren, A.F. Sadreev, A.A. Starikov, Nanotechnology 12, 562 (2001).

[18] K.-F. Berggren, A.F. Sadreev, A.A. Starikov, Phys. Rev. E 66, 016218 (2002).

[19] A.I. Saichev, H. Ishio, A.F. Sadreev, K.-F. Berggren, J. Phys. A 35, L87 (2002).

[20] K.J. Ebeling, Physical Acoustics: Principles, Methods, Eds. W.P Mason, R.N. Thurston, Vol. 17, Academic Press, New York 1984, p. 233.

[21] P.W. Brouwer, Phys. Rev. E 68, 046205 (2003).

[22] Y.-H. Kim, U. Kuhl, H.-J. Stöckmann, P.W. Brouwer, Phys. Rev. Lett. 94, 036804 (2005).

[23] A.F. Sadreev, K.-F. Berggren, Phys. Rev. E 70, 26201 (2004).

[24] O. Hul, S. Bauch, P. Pakoński, N. Savytskyy, K. Życzkowski, L. Sirko, Phys. Rev. E 69, 056205 (2005).

[25] P. Exner, P. Hejčik, P. Šeba, Acta Phys. Pol. A 109, 23 (2006).

[26] J.P. Bird, R. Akis, D.K. Ferry, A.P.S. de Moura, Y.C. Lai, K.M. Indlekofer, Rep. Prog. Phys. 66, 583 (2003).

[27] K.F. Berggren, I.I. Yakimenko, J. Hakanen, to be published.

[28] C.R. Paul, S.A. Nasar, L.E. Unnewehr, Introduction to Electrical Engineering, McGraw-Hill, Singapore 1992, ISBN 0-070-11322-X. 